Kurzbeiträge

Dieter Brenzke

\title{
Ein Lösungsvorschlag zur Klärung einer fragwürdigen Kalkulationsvorschrift in den Netzentgelt- verordnungen Strom und Gas
}

Eigener Lösungsvorschlag; Energierechtsreform 2005; fehlerhafter Lösungsvorschlag in einem „Positionspapier“; Netzregulierung; Unklarheit in den Vorschriften für die kalkulatorischen Kosten

Die Netzentgeltverordnungen Strom und Gas enthalten u.a. zwei Begriffe in zwei unterschiedlichen Vorschriften ( $\$ \$ 6$ und 7), welche sich in einem Zirkelschluss aufeinander beziehen. Aus dem Vorschriftentext geht nicht hervor, wie diese Größen (Betriebsnotwendiges Eigenkapital und Eigenkapitalquote) zu ermitteln sind. Inzwischen wurde von den Regulierungsbehörden ein Lösungsvorschlag verbreitet, der allerdings logisch nicht konsistent ist und zudem nach Auffassung des Verfassers gegen den Vorschriftentext selbst verstößt. Ein eigener Lösungsvorschlag vermag das Problem jedoch zu lösen.

\section{Darstellung der Fragwürdigkeit}

In beiden Verordnungen (Stromnetzentgeltverordnung StromNEV ${ }^{1}$ und Gasnetzentgeltverordnung $\mathrm{GasNEV}^{2}$ ) finden sich die folgenden gleich lautenden Kalkulationsvorschriften zur Kostenartenrechnung:

$\S 6$ Abs. 2 Satz 3 (Überschrift des $\S$ 6: „Kalkulatorische Abschreibungen“) lautet:

„Die Eigenkapitalquote ergibt sich rechnerisch als Quotient aus dem betriebsnotwendigen Eigenkapital und den kalkulatorisch ermittelten Restwerten des betriebsnotwendigen Vermögens zu historischen Anschaffungs- und Herstellungskosten.“

$\S 7$ Abs. 1 Satz 2 (Überschrift des $\S 7$, „Kalkulatorische Eigenkapitalverzinsung“) lautet:

„Das betriebsnotwendige Eigenkapital ergibt sich unter Berücksichtigung der Eigenkapitalquote nach $\S 6$ Abs. 2 ..."

Das heißt: Die Eigenkapitalquote gemäß $\S 6$ ist anhand des betriebsnotwendigen Eigenkapitals zu errechnen, das sich gemäß $\S 7$ unter Berücksichtigung der Eigenkapitalquote erst ergibt. Mit anderen Worten: In diesen beiden Vorschriften sind zwei unbekannte Größen enthalten, die sich gegenseitig bedingen. Allerdings findet sich im „Positionspa-

1 Vom 25. Juli 2005, BGB1 I Seite $2225 \mathrm{ff}$.

2 Vom 25. Juli 2005, BGBl I Seite $2197 \mathrm{ff}$. 
pier der Regulierungsbehörden des Bundes und der Länder zu Einzelfragen der Kostenkalkulation gemäß Stromnetzentgeltverordnung“(33 (im Folgenden: „Positionspapier") ein Lösungsansatz, der zunächst dargestellt und gewürdigt werden soll.

\section{Erster Ausweg: Das Positionspapier der Regulierungsbehörden}

\section{Darstellung des Lösungsweges}

Das Positionspapier ${ }^{4}$ beschreitet einen Ausweg, indem zwei Definitionen zweier Begriffe angenommen werden.

Erste Definition:

- Betriebsnotwendiges Vermögen I („BNV I“; definiert als Summe der kalkulatorischen Restwerte des Sachanlagevermögens zu historischen Anschaffungs- und Herstellungskosten plus Finanzanlagen plus Umlaufvermögen)

- Betriebsnotwendiges Eigenkapital I („BEK I“; definiert als BNV I minus Steueranteil des Sonderposten mit Rücklageanteil minus verzinsliches Fremdkapital minus Abzugskapital)

Die Eigenkapitalquote („EKQ“) ist der Quotient aus BEK I und BNV I.

Zweite Definition:

- Betriebsnotwendiges Vermögen II („BNV II“; definiert als kalkulatorische Restwerte des Sachanlagevermögens zu Tagesneuwerten mal Eigenkapitalquote; plus kalkulatorische Restwerte des Sachanlagevermögens zu historischen Anschaffungs- und Herstellungskosten mal Fremdkapitalquote; plus Finanzanlagen plus Umlaufvermögen)

- Betriebsnotwendiges Eigenkapital II („BEK II“; definiert als BNV II minus Steueranteil der Sonderposten mit Rücklageanteil minus verzinsliches Fremdkapital minus Abzugskapital).

Diese Vorgehensweise soll nun anhand angenommener - für einen Energieversorger mittlerer Größe jedoch plausibler und realistischer Zahlen - getestet werden.

\section{Test und Kritik}

Es werden folgende Zahlen (in Mio $€$ ) angenommen, wobei vereinfachend ausschließlich vom Vorhandensein von Altanlagen im Sinne von $\S 6$ Abs. 2 Sätze 1 und 2 Strom-/ GasNEV ausgegangen wird. In den folgenden Tabellen werden an Symbolen verwendet:

3 Stand: 7. März 2006, von der Bundesnetzagentur an die Verbände der Energiewirtschaft, unter anderem an den Verband Kommunaler Unternehmen in Köln, verteilt, von dort an dessen Mitglieder weiterverteilt.

4 Positionspapier Seiten 12 - 13 
RWAK: kalkulatorische Restwerte des Sachanlagevermögens zu historischen Anschaffungs- und Herstellungskosten

RWTNW: kalkulatorische Restwerte des Sachanlagevermögens zu Tagesneuwerten

FA: $\quad$ Finanzanlagen

UV: $\quad$ Umlaufvermögen

StASP: $\quad$ Steueranteil der Sonderposten mit Rücklageanteil

VFK: $\quad$ Verzinsliches Fremdkapital

AK: $\quad$ Abzugskapital (Rückstellungen, unverzinsliches Fremdkapital, Baukostenzuschüsse)

Summe FK: Summe aus StASP, VFK und AK.

Wie klar erkennbar ist, würde die Eigenkapitalquote (= BEK I / BNV I) zunächst 0,6370236 betragen. Das ist zu hoch, folglich ist der Höchstwert 0,4 (und entsprechend 0,6 für die Fremdkapitalquote) anzusetzen. Diese „Deckelung“ der Eigenkapitalquote geht aus $\S 6$ Abs. 2 Satz 4 Strom-/GasNEV hervor. Wendet man diesen Höchstwert an, nimmt man $\S 6$ Abs. 2 Satz 3 beim Wort und legt nun BEK II zu Grunde, ergäbe sich eine Eigenkapitalquote in Höhe von 0,7096189! Diese Abweichung kann nicht im Sinne des Gesetzgebers sein. Das Rechenbeispiel zeigt die Problematik der genannten Vorschrift auf eklatante Weise auf.

Die Frage ist natürlich, ob es - wie es das Positionspapier vorsieht - eine Doppeldefinition des betriebsnotwendigen Eigenkapitals - und damit zwangsläufig auch der Eigenkapitalquote -- geben darf. Der Verfasser ist der Meinung, dass dies nicht zulässig ist. Weiterhin muss bei diesem Test ausgeschlossen werden, dass diese eklatanten Abweichungen auf die „Deckelung“ (Eigenkapitalquote $=0,4$ ) zurückgeht. Daher sei nun mit einem auf erheblich höheren verzinslichen Fremdkapital gerechnet, um von vorneherein eine niedrigere Eigenkapitalquote zu erzielen (Daten wie in Tabelle 1, nur die Größe „VFK“ wurde von 3,5 auf 20 erhöht). 


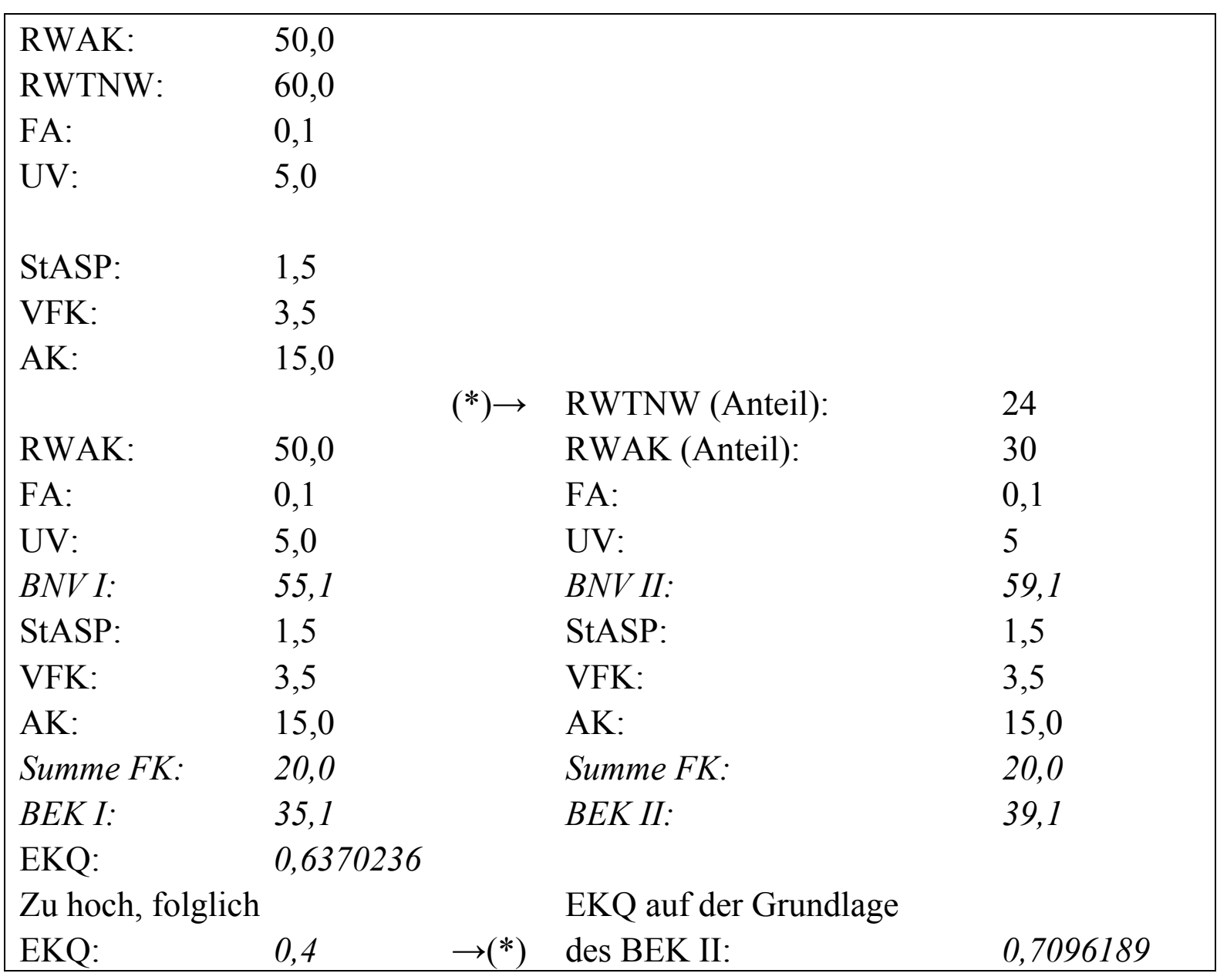

Tab. 1 Berechnung der Größen BNV I, BEK I, BNV II, BEK II und EKQ; erste Version Quelle: $\quad$ Eigene Darstellung

Auch bei dieser Version ergibt sich eine höhere gesetzeskonforme Eigenkapitalquote. Dieses Ergebnis zeigt, dass dieser Lösungsweg zu verwerfen ist, da er im Grunde gegen die genannten Vorschriften ( $\S 6$ und 7) verstößt. 


\begin{tabular}{|c|c|c|c|c|}
\hline VFK (neu): & 20,0 & & & \\
\hline & & $(*) \rightarrow$ & RWTNW (Anteil): & 20,3 \\
\hline RWAK: & 50,0 & & RWAK (Anteil): & 33,1 \\
\hline FA: & 0,1 & & FA: & 0,1 \\
\hline UV: & 5,0 & & UV: & 5,0 \\
\hline BNV I: & 55,1 & & BNV II: & 58,5 \\
\hline StASP: & 1,5 & & StASP: & 1,5 \\
\hline VFK: & 20,0 & & VFK: & 20,0 \\
\hline $\mathrm{AK}:$ & 15,0 & & AK: & 15,0 \\
\hline Summe FK: & 36,5 & & Summe FK: & 36,5 \\
\hline BEK I: & 18,6 & & BEK II: & 22,0 \\
\hline EKQ: & 0,3375681 & $\rightarrow(*)$ & & \\
\hline & & & $\begin{array}{l}\text { EKQ auf der Grundlage } \\
\text { des BEK II: }\end{array}$ & 0,3988327 \\
\hline
\end{tabular}

Tab. 2: Berechnung der Größen BNV I, BEK I, BNV II, BEK II und EKQ; zweite Version

Quelle: $\quad$ Eigene Darstellung

\section{Zweiter Ausweg: Eigener Lösungsvorschlag}

1. Darstellung des Lösungsweges

Im Grunde genommen ist die Lösung dieses Dilemmas einfach: Es geht um nichts anderes, als zwei Gleichungen mit zwei Unbekannten zu lösen. Diese lauten:

$\S 6$ Abs. 2 Satz 3:

(1) $\mathrm{EKQ}=\mathrm{BEK} /(\mathrm{RWAK}+\mathrm{FA}+\mathrm{UV})$

$\S 7$ Abs. 1 Satz 2:

(2) $\mathrm{BEK}=\mathrm{RWTNW} * \mathrm{EKQ}+\mathrm{RWAK} *(1-\mathrm{EKQ})+\mathrm{FA}+\mathrm{UV}-$ SummeFK

Löst man Gleichung (1) nach BEK auf und setzt beide Ausdrücke gleich, ergibt sich nach Umformung für

$$
\mathrm{EKQ}=(\mathrm{RWAK}+\mathrm{FA}+\mathrm{UV}-\mathrm{SummeFK}) /(2 * \mathrm{RWAK}-\mathrm{RWTNW}+\mathrm{FA}+\mathrm{UV})
$$

\section{Konsistenztest}

Ausgehend von den in Tabelle 2 verwendeten Zahlen ergibt sich nach der eben entwickelten Formel für die Eigenkapitalquote

$\mathrm{EKQ}=(50,0+0,1+5,0-41,5) /(2 * 50,0-60,0+0,1+5,0)=13,6 / 45,1=0,3015521$ (vgl. Tabelle 3, Daten wie in Tabelle 3). 


\begin{tabular}{|lll|}
\hline RWAK: & 34,9 & FKQ-Anteil \\
RWTNW: & 18,1 & EKQ-Anteil \\
FA: & 0,1 & \\
UV: & 5,0 & \\
BNV: & 58,1 & \\
StASP: & 1,5 & \\
AK: & 20,0 & \\
VFK: & 5,0 & \\
Summe FK: & 26,5 & \\
BEK: & 31,6 & \\
& EKQ: & 0,3015521 \\
\hline
\end{tabular}

Tab. 3: Berechnung der Eigenkapitalquote anhand der oben entwickelten Formel Quelle: $\quad$ Eigene Darstellung

Berechnet man die Eigenkapitalquote quasi zur Kontrolle noch einmal nach der Vorschrift des $\S 6$ Abs. 2 Satz 3 nach der dort festgelegten Anweisung, ergibt sich:

$$
\mathrm{EKQ}=\mathrm{BEK} /(\mathrm{RWAK}+\mathrm{FA}+\mathrm{UV})=16,6 / 55,1=0,3015521 .
$$

Man beachte, dass die nach der oben abgeleiteten Formel berechnete Größe für EKQ in die Größe BEK $=16,6$ eingeflossen ist $[$ RWAK $=34,9=50 *(1-0,3015521)$ und RWTNW $=18,1=60,0 * 0,3015521$ !]. Es ergibt sich ebenso wieder EKQ $=0,3015521$ - womit gezeigt ist, dass erstens die oben abgeleitete Formel korrekt ist und zudem dem Wortlaut des $\S 6$ Abs. 2 Satz 3 Rechnung trägt - quod erat demonstrandum!

Damit ist gezeigt, dass es unnötig ist, wie im „Positionspapier“ von jeweils zwei Größen für BNV und BEK auszugehen. Aber es bleibt die Frage, was geschieht, wenn sich für die Eigenkapitalquote ein Wert größer als 0,4 ergibt. Dies soll in der folgenden Fallvariante nachgeprüft werden (Tabelle 4). 


\begin{tabular}{|lll|}
\hline RWAK: & 30,0 & FKQ-Anteil \\
RWTNW: & 24,0 & EKQ-Anteil \\
FA: & 0,1 & \\
UV: & 5,0 & \\
BNV: & 59,1 & \\
StASP: & 1,5 & \\
AK: & 20,0 & \\
VFK: & 5,0 & \\
Summe FK: & $\mathbf{2 6 , 5}$ & \\
BEK: & 32,6 & \\
& EKQ: & 0,634146 \\
& Zu hoch; also: 0,4 \\
& EKQ nach § 6: 0,551607 \\
\hline
\end{tabular}

Tab. 4: Berechnung der Eigenkapitalquote mit „,Deckelung “

Quelle: $\quad$ Eigene Darstellung

Die Rechnung zeigt, dass die „Deckelung“ zu einer im Grunde zu hohen Eigenkapitalquote nach $\S 6$ führt. Aber in diesem Falle muss diese Abweichung der (zu hohen) rechnerischen Eigenkapitalquote von der ,gedeckelten“ hingenommen werden.

Dass sich bei der „Deckelung“ eine höhere Eigenkapitalquote nach $\S 6$ ergibt, ist unschädlich, denn es kommt auf die für die Berechnung der kalkulatorischen Abschreibungen und Eigenkapitalzinsen relevante Eigenkapitalquote an, weil eben das Ziel dieser Vorschrift die Bemessung dieser beiden Kostenarten und damit die Zubilligung eines bilanziellen Gewinns ist. Einen „Gleichklang“ von „gedeckelter“ Eigenkapitalquote und der nach $\S 6$ ermittelten kann es nicht geben, weil die Gleichung $0,4=(0,4 *$ RWTNW $+0,6 *$ RWAK + FA + UV - Summe FK $) /($ RWAK + FA + UV $)$ niemals aufgehen kann. Die EKQ ist ja primär Zielgröße, also Ergebnis einer Berechnung. Wird sie zur Prämisse gemacht (konstante, extern vorgegeben Größe), kann das oben dargestellte Gleichungssystem nicht mehr stimmen.

\section{Zusammenfassung}

Für welche Netzbetreiber ist der hier vorgestellte Lösungsweg zur rechtlich korrekten Ermittlung von Betriebsnotwendigem Eigenkapital und Eigenkapitalquote relevant? Grundsätzlich für alle, denn eine rechtlich korrekt ermittelte Eigenkapitalquote ist Maßstab dafür, ob die „Deckelung“ auf 0,4 zu erfolgen hat. Muss „gedeckelt“ werden, ist die Berechnung des nunmehr modifizierten Betriebsnotwendigen Eigenkapitals unproblematisch. Am bedeutsamsten ist die korrekte Ermittlung dieser beiden Größen v.a. für all diejenigen Netzbetreiber, deren Eigenkapitalquote unter 0,4 liegt. 